\title{
Hyoglossus Muscle
}

National Cancer Institute

\section{Source}

National Cancer Institute. Hyoglossus Muscle. NCI Thesaurus. Code C53161.

A muscle extending from the hyoid bone to the side of the tongue that retracts and pulls the side of the tongue downward. 